\title{
Measurement of the Charged-Pion Polarizability
}

C. Adolph, ${ }^{8}$ R. Akhunzyanov, ${ }^{7}$ M. G. Alexeev, ${ }^{27}$ G. D. Alexeev, ${ }^{7}$ A. Amoroso, ${ }^{27,29}$ V. Andrieux,${ }^{22}$ V. Anosov, ${ }^{7}$ A. Austregesilo, ${ }^{10,17}$ B. Badełek, ${ }^{31}$ F. Balestra, ${ }^{27,29}$ J. Barth, ${ }^{4}$ G. Baum, ${ }^{1}$ R. Beck, ${ }^{3}$ Y. Bedfer, ${ }^{22}$ A. Berlin, ${ }^{2}$ J. Bernhard,${ }^{13}$ K. Bicker, ${ }^{10,17}$ J. Bieling, ${ }^{4}$ R. Birsa, ${ }^{25}$ J. Bisplinghoff, ${ }^{3}$ M. Bodlak,${ }^{19}$ M. Boer, ${ }^{22}$ P. Bordalo, ${ }^{12, \dagger}$ F. Bradamante, ${ }^{24,25}$ C. Braun, ${ }^{8}$ A. Bressan, ${ }^{24,25}$ M. Büchele, ${ }^{9}$ E. Burtin, ${ }^{22}$ L. Capozza, ${ }^{22}$ M. Chiosso, ${ }^{27,29}$ S. U. Chung, ${ }^{17}$ A. Cicuttin, ${ }^{26,25}$ M. Colantoni, ${ }^{29}$ M. L. Crespo, ${ }^{26,25}$ Q. Curiel, ${ }^{22}$ S. Dalla Torre, ${ }^{25}$ S. S. Dasgupta, ${ }^{6}$ S. Dasgupta, ${ }^{25}$ O. Yu. Denisov, ${ }^{29}$ A. M. Dinkelbach, ${ }^{17}$ S. V. Donskov, ${ }^{21}$ N. Doshita, ${ }^{33}$ V. Duic, ${ }^{24}$ W. Dünnweber, ${ }^{16}$ M. Dziewiecki, ${ }^{32}$ A. Efremov, ${ }^{7}$ C. Elia, ${ }^{24,25}$ P. D. Eversheim, ${ }^{3}$ W. Eyrich, ${ }^{8}$ M. Faessler, ${ }^{16}$ A. Ferrero, ${ }^{22}$ A. Filin, ${ }^{21}$ M. Finger, ${ }^{19}$ M. Finger, Jr., ${ }^{19}$ H. Fischer, ${ }^{9}$ C. Franco,${ }^{12}$ N. du Fresne von Hohenesche, ${ }^{13,10}$ J. M. Friedrich, ${ }^{17}$ V. Frolov ${ }^{10}$ F. Gautheron, ${ }^{2}$ O. P. Gavrichtchouk, ${ }^{7}$ S. Gerassimov, ${ }^{15,17}$

R. Geyer, ${ }^{16}$ I. Gnesi, ${ }^{27,29}$ B. Gobbo, ${ }^{25}$ S. Goertz, ${ }^{4}$ M. Gorzellik, ${ }^{9}$ S. Grabmüller, ${ }^{17}$ A. Grasso, ${ }^{27,29}$ B. Grube, ${ }^{17}$

T. Grussenmeyer, ${ }^{9}$ A. Guskov, ${ }^{7}$ T. Guthörl,,${ }^{9}$ F. Haas, ${ }^{17}$ D. von Harrach, ${ }^{13}$ D. Hahne, ${ }^{4}$ R. Hashimoto, ${ }^{33}$ F. H. Heinsius, ${ }^{9}$ F. Herrmann, ${ }^{9}$ F. Hinterberger, ${ }^{3}$ Ch. Höppner, ${ }^{17}$ N. Horikawa, ${ }^{18,8}$ N. d'Hose, ${ }^{22}$ S. Huber, ${ }^{17}$ S. Ishimoto, ${ }^{33, \|}$ A. Ivanov, ${ }^{7}$ Yu. Ivanshin, ${ }^{7}$ T. Iwata, ${ }^{33}$ R. Jahn, ${ }^{3}$ V. Jary, ${ }^{20}$ P. Jasinski, ${ }^{13}$ P. Jörg, ${ }^{9}$ R. Joosten, ${ }^{3}$ E. Kabuß, ${ }^{13}$ B. Ketzer, ${ }^{17,}$ G. V. Khaustov, ${ }^{21}$ Yu. A. Khokhlov, ${ }^{21, * *}$ Yu. Kisselev, ${ }^{7}$ F. Klein, ${ }^{4}$ K. Klimaszewski, ${ }^{30}$ J. H. Koivuniemi, ${ }^{2}$ V. N. Kolosov, ${ }^{21}$ K. Kondo, ${ }^{33}$

K. Königsmann, ${ }^{9}$ I. Konorov, ${ }^{15,17}$ V. F. Konstantinov, ${ }^{21}$ A. M. Kotzinian, ${ }^{27,29}$ O. Kouznetsov, ${ }^{7}$ M. Krämer, ${ }^{17}$ Z.

V. Kroumchtein, ${ }^{7}$ N. Kuchinski, ${ }^{7}$ R. Kuhn, ${ }^{17}$ F. Kunne, ${ }^{22}$ K. Kurek,${ }^{30}$ R. P. Kurjata, ${ }^{32}$ A. A. Lednev, ${ }^{21}$ A. Lehmann, ${ }^{8}$ M. Levillain ${ }^{22}$ S. Levorato,${ }^{25}$ J. Lichtenstadt, ${ }^{23}$ A. Maggiora, ${ }^{29}$ A. Magnon, ${ }^{22}$ N. Makke, ${ }^{24,25}$ G. K. Mallot,${ }^{10}$ C. Marchand, ${ }^{22}$ A. Martin, ${ }^{24,25}$ J. Marzec, ${ }^{32}$ J. Matousek, ${ }^{19}$ H. Matsuda, ${ }^{33}$ T. Matsuda, ${ }^{14}$ G. Meshcheryakov, ${ }^{7}$ W. Meyer, ${ }^{2}$ T. Michigami, ${ }^{33}$ Yu. V. Mikhailov, ${ }^{21}$ Y. Miyachi, ${ }^{33}$ M. A. Moinester, ${ }^{23}$ A. Nagaytsev, ${ }^{7}$ T. Nagel, ${ }^{17}$ F. Nerling, ${ }^{13}$ S. Neubert, ${ }^{17}$ D. Neyret, ${ }^{22}$ V. I. Nikolaenko, ${ }^{21}$ J. Novy, ${ }^{20}$ W. -D. Nowak, ${ }^{9}$ A. S. Nunes, ${ }^{12}$ A. G. Olshevsky, ${ }^{7}$ I. Orlov, ${ }^{7}$ M. Ostrick, ${ }^{13}$ R. Panknin, ${ }^{4}$ D. Panzieri, ${ }^{28,29}$ B. Parsamyan, ${ }^{27,29}$ S. Paul, ${ }^{17}$ D. Peshekhonov, ${ }^{7}$ S. Platchkov, ${ }^{22}$ J. Pochodzalla, ${ }^{13}$ V. A. Polyakov, ${ }^{21}$ J. Pretz, ${ }^{4, \dagger}$ M. Quaresma, ${ }^{12}$ C. Quintans, ${ }^{12}$ S. Ramos, ${ }^{12, \dagger}$ C. Regali, ${ }^{9}$ G. Reicherz, ${ }^{2}$ E. Rocco, ${ }^{10}$ N. S. Rossiyskaya, ${ }^{7}$ D. I. Ryabchikov, ${ }^{21}$ A. Rychter, ${ }^{32}$ V. D. Samoylenko, ${ }^{21}$ A. Sandacz, ${ }^{30}$ S. Sarkar, ${ }^{6}$ I. A. Savin, ${ }^{7}$ G. Sbrizzai, ${ }^{24,25}$

P. Schiavon, ${ }^{24,25}$ C. Schill,,${ }^{9}$ T. Schlüter, ${ }^{16}$ K. Schmidt,,${ }^{9}$ H. Schmieden, ${ }^{4}$ K. Schönning, ${ }^{10}$ S. Schopferer, ${ }^{9}$ M. Schott, ${ }^{10}$ O. Yu. Shevchenko, ${ }^{7, *}$ L. Silva, ${ }^{12}$ L. Sinha, ${ }^{6}$ S. Sirtl, ${ }^{9}$ M. Slunecka ${ }^{7}$ S. Sosio, ${ }^{27,29}$ F. Sozzi, ${ }^{25}$ A. Srnka, ${ }^{5}$ L. Steiger, ${ }^{25}$ M. Stolarski, ${ }^{12}$ M. Sulc, ${ }^{11}$ R. Sulej, ${ }^{30}$ H. Suzuki, ${ }^{33,8}$ A. Szabelski, ${ }^{30}$ T. Szameitat, ${ }^{9, *}$ P. Sznajder, ${ }^{30}$ S. Takekawa, ${ }^{27,29}$ J. ter Wolbeek, ${ }^{9,}$ S. Tessaro, ${ }^{25}$ F. Tessarotto, ${ }^{25}$ F. Thibaud ${ }^{22}$ S. Uhl, ${ }^{17}$ I. Uman, ${ }^{16}$ M. Virius, ${ }^{20}$ L. Wang, ${ }^{2}$ T. Weisrock, ${ }^{13}$ M. Wilfert, ${ }^{13}$ R. Windmolders, ${ }^{4} \mathrm{H}$. Wollny, ${ }^{22} \mathrm{~K}$. Zaremba, ${ }^{32} \mathrm{M}$. Zavertyaev, ${ }^{15}$ E. Zemlyanichkina, ${ }^{7}$ M. Ziembicki, ${ }^{32}$ and A. Zink $^{8}$

\section{(COMPASS Collaboration)}

\author{
${ }^{1}$ Universität Bielefeld, Fakultät für Physik, 33501 Bielefeld, Germany \\ ${ }^{2}$ Universität Bochum, Institut für Experimentalphysik, 44780 Bochum, Germany \\ ${ }^{3}$ Universität Bonn, Helmholtz-Institut für Strahlen- und Kernphysik, 53115 Bonn, Germany \\ ${ }^{4}$ Universität Bonn, Physikalisches Institut, 53115 Bonn, Germany \\ ${ }^{5}$ Institute of Scientific Instruments, AS CR, 61264 Brno, Czech Republic \\ ${ }^{6}$ Matrivani Institute of Experimental Research \& Education, Calcutta 700 030, India \\ ${ }^{7}$ Joint Institute for Nuclear Research, 141980 Dubna, Moscow Region, Russia \\ ${ }^{8}$ Universität Erlangen-Nürnberg, Physikalisches Institut, 91054 Erlangen, Germany \\ ${ }^{9}$ Universität Freiburg, Physikalisches Institut, 79104 Freiburg, Germany \\ ${ }^{10}$ CERN, 1211 Geneva 23, Switzerland \\ ${ }^{11}$ Technical University in Liberec, 46117 Liberec, Czech Republic \\ ${ }^{12}$ LIP, 1000-149 Lisbon, Portugal \\ ${ }^{13}$ Universität Mainz, Institut für Kernphysik, 55099 Mainz, Germany \\ ${ }^{14}$ University of Miyazaki, Miyazaki 889-2192, Japan \\ ${ }^{15}$ Lebedev Physical Institute, 119991 Moscow, Russia \\ ${ }^{16}$ Ludwig-Maximilians-Universität München, Department für Physik, 80799 Munich, Germany \\ ${ }^{17}$ Technische Universität München, Physik Department, 85748 Garching, Germany \\ ${ }^{18}$ Nagoya University, 464 Nagoya, Japan \\ ${ }^{19}$ Charles University in Prague, Faculty of Mathematics and Physics, 18000 Prague, Czech Republic \\ ${ }^{20}$ Czech Technical University in Prague, 16636 Prague, Czech Republic
}


${ }^{21}$ State Scientific Center Institute for High Energy Physics of National Research Center “Kurchatov Institute,” 142281 Protvino, Russia

${ }^{22}$ CEA IRFU/SPhN Saclay, 91191 Gif-sur-Yvette, France

${ }^{23}$ Tel Aviv University, School of Physics and Astronomy, 69978 Tel Aviv, Israel

${ }^{24}$ University of Trieste, Department of Physics, 34127 Trieste, Italy

${ }^{25}$ Trieste Section of INFN, 34127 Trieste, Italy

${ }^{26}$ Abdus Salam ICTP, 34151 Trieste, Italy

${ }^{27}$ University of Turin, Department of Physics, 10125 Turin, Italy

${ }^{28}$ University of Eastern Piedmont, 15100 Alessandria, Italy

${ }^{29}$ Torino Section of INFN, 10125 Turin, Italy

${ }^{30}$ National Centre for Nuclear Research, 00-681 Warsaw, Poland

${ }^{31}$ University of Warsaw, Faculty of Physics, 00-681 Warsaw, Poland

${ }^{32}$ Warsaw University of Technology, Institute of Radioelectronics, 00-665 Warsaw, Poland

${ }^{33}$ Yamagata University, Yamagata, 992-8510 Japan

(Received 2 June 2014; revised manuscript received 24 December 2014; published 10 February 2015)

The COMPASS collaboration at CERN has investigated pion Compton scattering, $\pi^{-} \gamma \rightarrow \pi^{-} \gamma$, at centerof-mass energy below 3.5 pion masses. The process is embedded in the reaction $\pi^{-} \mathrm{Ni} \rightarrow \pi^{-} \gamma \mathrm{Ni}$, which is initiated by $190 \mathrm{GeV}$ pions impinging on a nickel target. The exchange of quasireal photons is selected by isolating the sharp Coulomb peak observed at smallest momentum transfers, $Q^{2}<0.0015(\mathrm{GeV} / c)^{2}$. From a sample of 63000 events, the pion electric polarizability is determined to be $\alpha_{\pi}=\left(2.0 \pm 0.6_{\text {stat }} \pm\right.$ $\left.0.7_{\text {syst }}\right) \times 10^{-4} \mathrm{fm}^{3}$ under the assumption $\alpha_{\pi}=-\beta_{\pi}$, which relates the electric and magnetic dipole polarizabilities. It is the most precise measurement of this fundamental low-energy parameter of strong interaction that has been addressed since long by various methods with conflicting outcomes. While this result is in tension with previous dedicated measurements, it is found in agreement with the expectation from chiral perturbation theory. An additional measurement replacing pions by muons, for which the crosssection behavior is unambiguously known, was performed for an independent estimate of the systematic uncertainty.

DOI: 10.1103/PhysRevLett.114.062002

The electric and magnetic polarizabilities of an extended object describe its rigidity against deformation by external electric and magnetic fields, respectively. For a strongly interacting particle, the polarizabilities are of special interest as they are related to the inner forces determining the substructure and, thus, provide valuable information about quantum chromodynamics (QCD) at low energy. The pion is of specific interest in that regard, as it represents the lightest QCD bound state, and its polarizability, once experimentally determined, imposes stringent constraints on theory as discussed below.

For the proton, the polarizability is measured directly via Compton scattering on a hydrogen target. In contrast, for charged pions the experimental situation is more difficult since they are not available as fixed targets. Although different techniques exist, all previous measurements are affected by large experimental and theoretical uncertainties, see, e.g., Refs. [1-3]. Groundbreaking work at Serpukhov [1] employed the same Primakoff technique [4] as used in

Published by the American Physical Society under the terms of the Creative Commons Attribution 3.0 License. Further distribution of this work must maintain attribution to the author(s) and the published article's title, journal citation, and DOI.
PACS numbers: 13.60.-r, 12.39.Fe, 13.60.Le, 25.80.Dj

this Letter; however, low statistics made it difficult, at that time, to evaluate the systematic uncertainty.

The electric and magnetic dipole polarizabilities $\alpha_{\pi}$ and $\beta_{\pi}$ appear at the level of the pion Compton cross section $\sigma_{\pi \gamma}$ for the reaction $\pi^{-} \gamma \rightarrow \pi^{-} \gamma$ in the correction to the Born cross section for the pointlike particle at linear order $[5,6]$ as

$$
\begin{aligned}
\frac{d \sigma_{\pi \gamma}}{d \Omega}= & \left(\frac{d \sigma_{\pi \gamma}}{d \Omega}\right)_{\text {Born }}-\frac{\alpha m_{\pi}^{3}\left(s-m_{\pi}^{2}\right)^{2}}{4 s^{2}\left(s z_{+}+m_{\pi}^{2} z_{-}\right)} \\
& \times\left(z_{-}^{2}\left(\alpha_{\pi}-\beta_{\pi}\right)+\frac{s^{2}}{m_{\pi}^{4}} z_{+}^{2}\left(\alpha_{\pi}+\beta_{\pi}\right)\right) .
\end{aligned}
$$

Here, $\alpha \approx 1 / 137.04$ is the fine structure constant, $z_{ \pm}=$ $1 \pm \cos \theta_{\mathrm{cm}}$ with $\theta_{\mathrm{cm}}$ being the $\pi \gamma$ scattering angle, $s$ is the squared total energy in the center-of-mass reference frame, and $m_{\pi}$ is the rest mass of the charged pion. Higher-order contributions can be parametrized by further multipole polarizabilities, which are neglected in this analysis.

For hadronic interactions at low energy, QCD can be formulated in terms of an effective field theory that results from the systematic treatment of chiral symmetry and its breaking pattern, which is called chiral perturbation theory (ChPT). In this approach, the pions $\left(\pi^{+}, \pi^{0}, \pi^{-}\right)$are identified with the Goldstone bosons associated with 
spontaneous chiral symmetry breaking. Properties and interactions of pions, hence, provide the most rigorous test regarding whether ChPT is the correct low-energy representation of QCD. The predictions for the dynamics of low-energy $\pi \pi$ scattering were confirmed in various experiments, see, e.g., Ref. [7]. However, in the case of $\pi \gamma$ scattering, the "Serpukhov value" $\alpha_{\pi}=(6.8 \pm 1.8) \times$ $10^{-4} \mathrm{fm}^{3}$ [1] for the pion polarizability deviates from the ChPT prediction $\alpha_{\pi}=(2.9 \pm 0.5) \times 10^{-4} \mathrm{fm}^{3}$ [8]. This observation, which was confirmed in radiative pion photoproduction at MAMI [2], remained unexplained for more than two decades.

In pion-nucleus reactions, photon exchange becomes important at very low momentum transfer and competes with strong interaction processes. The $\pi$-nucleus cross section can be connected to the $\pi \gamma$ cross section using the equivalent-photon approximation (EPA) [9]

$$
\frac{d \sigma_{(A, Z)}^{\mathrm{EPA}}}{d s d Q^{2} d \Phi_{n}}=\frac{Z^{2} \alpha}{\pi\left(s-m_{\pi}^{2}\right)} F^{2}\left(Q^{2}\right) \frac{Q^{2}-Q_{\min }^{2}}{Q^{4}} \frac{d \sigma_{\pi \gamma \rightarrow X}}{d \Phi_{n}} .
$$

Here, the cross section for the process $\pi^{-}(A, Z) \rightarrow$ $X^{-}(A, Z)$ is factorized into the quasireal photon density provided by the nucleus of charge $Z$, and $\sigma_{\pi \gamma \rightarrow X}$ denotes the cross section for the embedded $\pi^{-} \gamma \rightarrow X^{-}$reaction of a pion and a real photon. The function $F\left(Q^{2}\right)$ is the electromagnetic form factor of the nucleus and $d \Phi_{n}$ is the $n$-particle phase-space element of the final-state system $X^{-}$. The minimum value of the negative 4-momenta transfer squared, $Q^{2}=-\left(p_{\text {beam }}^{\mu}-p_{X}^{\mu}\right)^{2}$, is $Q_{\min }^{2}=\left(s-m_{\pi}^{2}\right)^{2} /$ $\left(4 E_{\text {beam }}^{2}\right)$ for a given final-state mass $m_{X}=\sqrt{s}$, with typical values $Q_{\min }^{2}=(1 \mathrm{MeV} / c)^{2}$. In the analysis presented in this Letter, the observed final state is $\pi^{-} \gamma$, and the investigated cross section $\sigma_{\pi \gamma \rightarrow X}$ is $\sigma_{\pi \gamma}$ as introduced along with Eq. (1) with $s=\left(p_{\pi}^{\mu}+p_{\gamma}^{\mu}\right)^{2}$ being determined by the four-vectors of the two outgoing particles. The same experimental technique has been employed previously at COMPASS for the $\pi^{-} \pi^{-} \pi^{+}$final state [7].

The COMPASS experiment [10] is situated at the M2 beam line of the CERN Super Proton Synchrotron. For this measurement, negative muons or hadrons of $190 \mathrm{GeV} / c$ were used, which were impinging on a $4 \mathrm{~mm}$ thick nickel target. The hadronic components of the hadron beam at the target position are $96.8 \% \pi^{-}, 2.4 \% \mathrm{~K}^{-}$, and $0.8 \% \bar{p}$. The hadron beam also contains about $1 \%$ of muons and a small amount of electrons. The pions are identified with a Cherenkov counter located in the beam line at the entrance to the experimental area. The large-acceptance highprecision spectrometer is well suited for investigations of high-energy reactions at low to intermediate momentum transfer to the target nucleus. Outgoing charged particles are detected by the tracking system and their momenta are determined using two large aperture magnets. Tracks crossing more than 15 radiation lengths equivalent thickness of material are treated as muons. The small-angle electromagnetic calorimeter ECAL2 detects photons up to scattering angles of about $40 \mathrm{mrad}$.

The data presented in this Letter were recorded in the year 2009 using, alternatively, either hadron or muon beams. The trigger logic selects events with an energy deposit of more than $70 \mathrm{GeV}$ in the central part of ECAL2 in coincidence with an incoming beam particle. In the data analysis, exactly one scattered, negatively charged particle, which is assumed to be a pion, is required to form, with the incoming pion, a vertex that is consistent with an interaction in the target volume. Exactly one cluster in ECAL2 with an energy above $2 \mathrm{GeV}$, which is not attributed to a produced charged particle, is required and taken as the produced photon. In order to avoid the kinematic region that is dominated by multiple scattering of the outgoing pion in the target material, only events with $p_{T}>$ $40 \mathrm{MeV} / c$ are accepted, as shown in Fig. 1(a). This cut also removes contributions of the reaction $e^{-} \mathrm{Ni} \rightarrow e^{-} \gamma \mathrm{Ni}$. Neglecting the tiny recoil of the target nucleus at low $Q^{2}$, the sum of the scattered pion energy $E_{\pi}$ and the photon energy $E_{\gamma}$ equals the beam energy for the exclusive reaction $\pi^{-} \mathrm{Ni} \rightarrow \pi^{-} \gamma \mathrm{Ni}$. The distribution of events as a function of the energy balance $\Delta E=E_{\pi}+E_{\gamma}-E_{\text {beam }}$ is presented in Fig. 1(b). As the calorimetric energy resolution is approximately constant over the range of interest and about $3 \mathrm{GeV}$, the energy balance is required to be $|\Delta E|<15 \mathrm{GeV}$. After this selection, we assume the reaction $\pi^{-} \mathrm{Ni} \rightarrow \pi^{-} \gamma \mathrm{Ni}$ and imposing energy conservation, we rescale the photon momentum vector such that $E_{\gamma}=E_{\text {beam }}-E_{\pi}$, as the photon energy is the least known quantity. The distribution of events as a function of $|Q|=\sqrt{Q^{2}}$ is given in Fig. 1(c). The peak width of about $12 \mathrm{MeV} / c$ is dominated by the experimental resolution, which is about a factor of 10 larger than the true width of the Coulomb distribution. Events corresponding to photon exchange are selected by requiring $Q^{2}<$ $0.0015(\mathrm{GeV} / c)^{2}$. The size of the Coulomb peak was checked for different targets on smaller-statistics data (tungsten, silicon, carbon), showing consistency with the approximate $\sim Z^{2}$ expectation. Background contributions from intermediate $\rho^{-}(770)$ production with decay into $\pi^{-} \pi^{0}$ are suppressed by restricting to the mass interval $m_{\pi \gamma}<$ $3.5 m_{\pi} \approx 0.487 \mathrm{GeV} / c^{2}$, as shown in Fig. 1(d). For this analysis, we choose the region $0.4<x_{\gamma}<0.9$, where $x_{\gamma}=$ $E_{\gamma} / E_{\text {beam }}$ is the fraction of the beam energy taken by the photon in the laboratory system. This region is characterized by constant trigger efficiency and effective identification of muons. The number of $\pi \gamma$ events in this region is 63000 .

The pion polarizability manifests itself by a modification of the differential Compton cross section at high photon energies that correspond to large forces exerted to the pion. For retrieving the pion polarizability from the shape of the measured cross section, the analysis technique as described in Ref. [1] is adopted. This includes the assumption that $\alpha_{\pi}$ is approximately equal in magnitude to the magnetic 


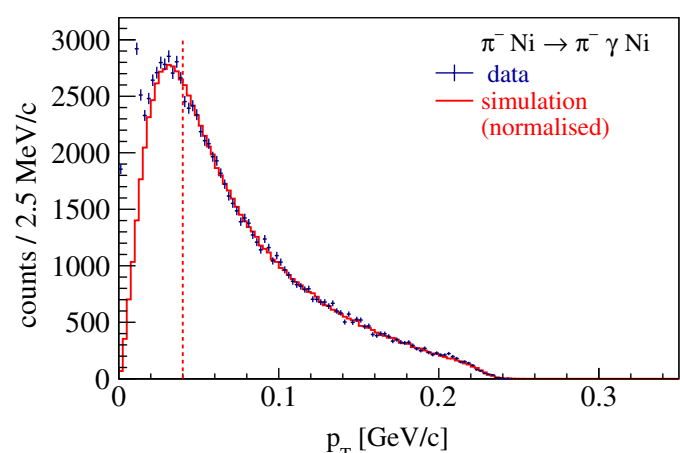

(a)

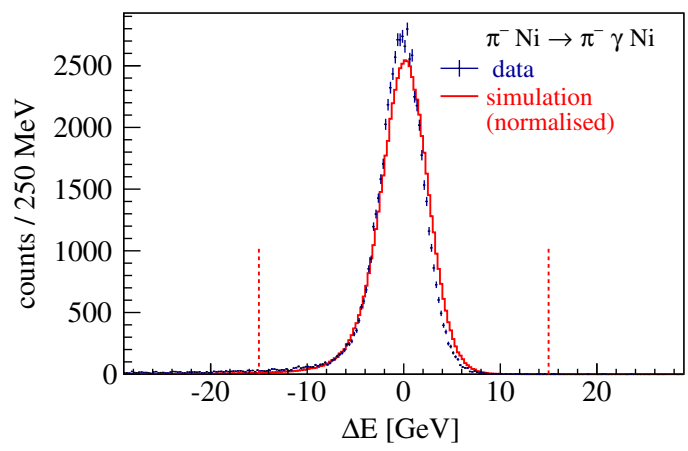

(b)

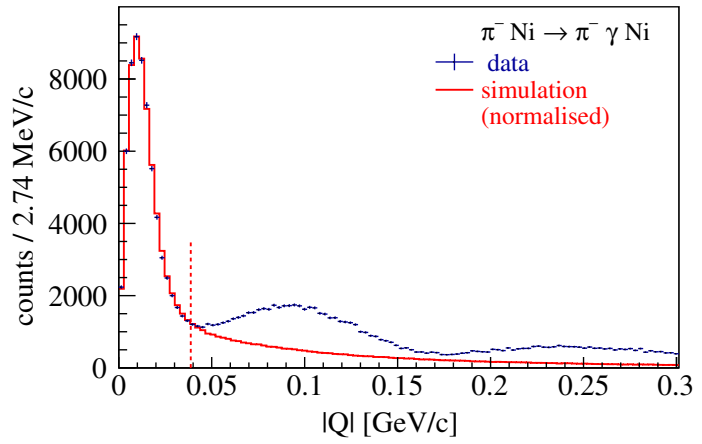

(c)

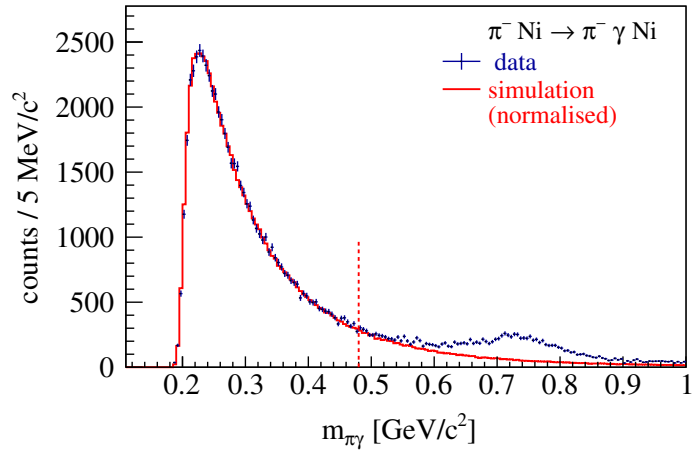

(d)

FIG. 1 (color online). Comparison of the measured (black points with error bars) and simulated (red histograms) kinematic distributions for measurements with pion beam: (a) transverse momentum $p_{T}$ of the scattered pion; (b) energy balance $\Delta E$; (c) $|Q|$ distribution, featuring for the real data at higher values the contribution from strong interaction, which is not contained in the simulation; (d) invariant mass of the $\pi \gamma$ system. The dotted lines indicate the cuts as explained in the text. polarizability $\beta_{\pi}$, but with opposite sign. In this analysis, we use $\alpha_{\pi}=-\beta_{\pi}$. The polarizability is determined from the $x_{\gamma}$ dependence of the ratio

$$
R_{\pi}=\left(\frac{d \sigma_{\pi \gamma}}{d x_{\gamma}}\right) /\left(\frac{d \sigma_{\pi \gamma}^{0}}{d x_{\gamma}}\right)=1-\frac{3}{2} \frac{m_{\pi}^{3}}{\alpha} \frac{x_{\gamma}^{2}}{1-x_{\gamma}} \alpha_{\pi},
$$

where $\sigma_{\pi \gamma}=N / L$ refers to the measured cross section, $d \sigma_{\pi \gamma}^{0}$ to the simulated cross section expected for $\alpha_{\pi}=0$ (including corrections to the pure Born cross section as those from chiral loops, as specified below), $N$ is the number of events, and $L$ is the integrated luminosity. The variable $x_{\gamma}$ is, to a good approximation, related to the photon scattering angle by $\cos \theta_{\mathrm{cm}} \approx 1-2 x_{\gamma} /\left(1-m_{\pi}^{2} / s\right)$, so that the selected range in $x_{\gamma}$ corresponds to $-1<\cos \theta_{\mathrm{cm}}<0.15$, where the sensitivity to $\alpha_{\pi}-\beta_{\pi}$ is largest, see Eq. (1). The event distribution in the variable $x_{\gamma}$ is shown in Fig. 2 together with the simulated data that were generated with $\alpha_{\pi}=0$ and scaled such that the integral is the same as for the real data, disregarding, at this point, the small effect of the pion polarizability. The requirement $\Delta E<15 \mathrm{GeV}$ and the observation of exactly one photon in ECAL2 do not completely eliminate the background from $\pi^{0}$ mesons produced in electromagnetic and strong interactions, $\pi^{-} \mathrm{Ni} \rightarrow \pi^{-} \pi^{0} X$, where, in the considered low $Q^{2}$ region, $X$ is predominantly a Ni nucleus in its ground state, but in principle, nuclear excitation or breakup is also included. The probability to misidentify such $\pi^{-} \pi^{0}$ events as $\pi^{-} \gamma$ events due to missing or overlapping photons is estimated from a pure sample of beam kaon decays, $K^{-} \rightarrow \pi^{-} \pi^{0}$, and the observation of corresponding (in this case unphysical) $\pi^{-} \gamma$ final states. The same probability is assumed for misidentifying $\pi^{-} \pi^{0}$ as $\pi^{-} \gamma$ for the studied $\pi^{-} \mathrm{Ni}$ reactions in each $x_{\gamma}$ bin, and the fraction $f_{\pi^{0}}$ of

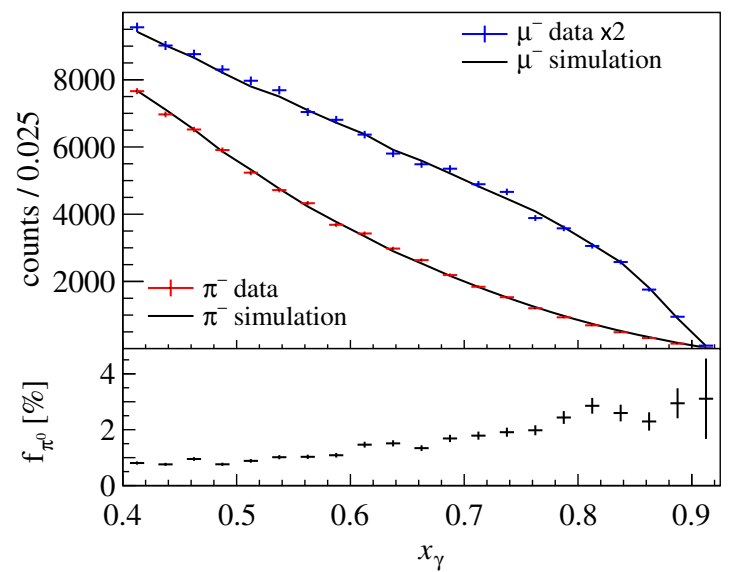

FIG. 2 (color online). The measured and simulated $x_{\gamma}$ distributions for pion (lower curve) and muon (upper curve) beam. The statistical uncertainty of the real data points is indicated by vertical error bars, while the width of the symbols is set arbitrarily to one third of the bin width. The lines connect the simulation results for the same bin centers. The bottom panel shows the $\pi^{0}$ background fraction $f_{\pi^{0}}$ that was subtracted from the pion data. 
background caused by $\pi^{0}$ events is presented in the bottom panel of Fig. 2 as a function of $x_{\gamma}$. The simulated cross section $d \sigma_{\pi \gamma}^{0} / d x_{\gamma}$ contains, besides the Born term, the following corrections: (i) radiative corrections [11]; (ii) chiral loop corrections [12]; (iii) corrections for the electromagnetic form factor of the nickel nucleus, which is approximated for simplicity by the equivalent sharp-radius formula $F\left(Q^{2}\right)=j_{1}(r q)$ with $r=5.0 \mathrm{fm}$, where $q$ is the modulus of the 3-momenta transfer to the nucleus. More precise form-factor parametrizations were checked with no visible influence on the results. These corrections influence the $x_{\gamma}$ spectrum such that the extracted polarizability is increased by $0.6 \times 10^{-4} \mathrm{fm}^{3}$ after they are applied. The ratio of the measured differential cross section $d \sigma_{\pi \gamma} / d x_{\gamma}$ to the expected cross section for a pointlike spin-0 particle taken from the simulation is shown in the top panel of Fig. 3. The fit of the ratio $R_{\pi}$ by Eq. (3) in the range $0.4<x_{\gamma}<0.9$, using the integrated luminosity $L$ as additional free parameter, yields the pion polarizability: $\alpha_{\pi}=\left(2.0 \pm 0.6_{\text {stat }}\right) \times 10^{-4} \mathrm{fm}^{3}$.

The systematic uncertainty of the measurement, as summarized in Table I, accounts for: (i) uncertainty of

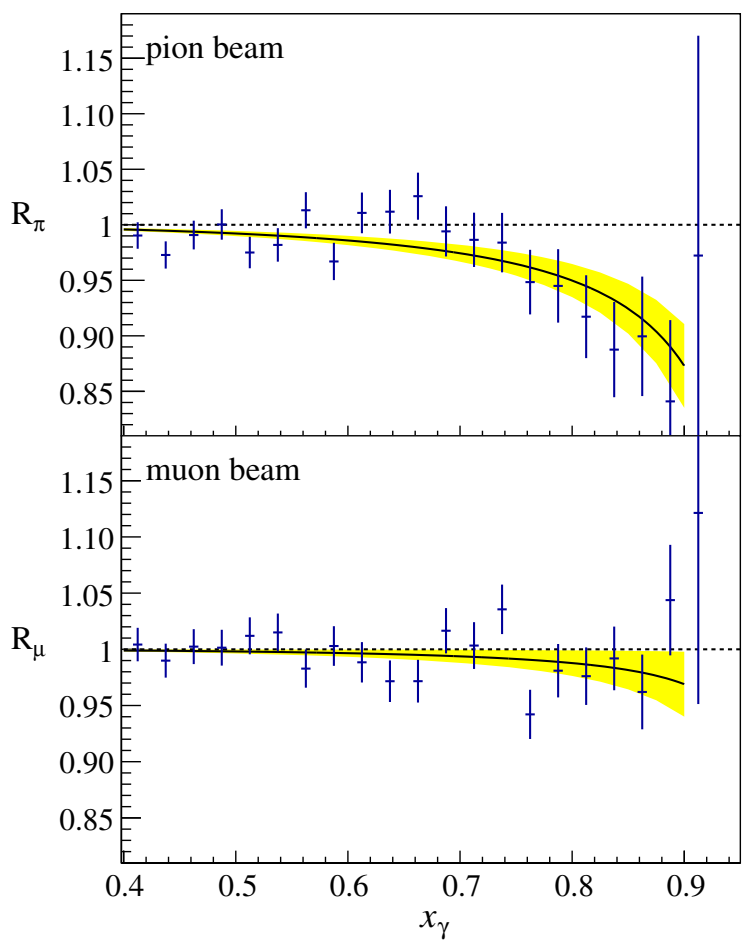

FIG. 3 (color online). The $x_{\gamma}$ dependence of the ratio of the measured differential cross section $d \sigma / d x_{\gamma}$ over the expected cross section for pointlike particles. Top (bottom) panel: measurement with pion (muon) beam. The respective ratios contain the corrections described in the text. The bands denote the respective statistical uncertainties of the fit results shown by the solid lines. Error bars denote statistical uncertainties. The quality of the fits can be characterized by the values $\chi_{\pi}^{2} / \mathrm{NDF}=22.0 / 18$ and $\chi_{\mu}^{2} / \mathrm{NDF}=19.6 / 18$, respectively. the determination of the tracking detector efficiency for the simulation; (ii) uncertainty related to the neglect of Coulomb corrections [13] and of corrections for nuclear charge screening by atomic electrons and for multiplephoton exchange [14]; (iii) statistical uncertainty of the $\pi^{0}$ background subtraction; (iv) effect of the uncertainty on the estimate of strong interaction background and its interference with the Coulomb contribution; (v) contribution from the elastic pion-electron scattering process; (vi) contribution from the $\mu^{-} \mathrm{Ni} \rightarrow \mu^{-} \gamma \mathrm{Ni}$ reaction, where the scattered muon was misidentified as a pion. The total systematic uncertainty is obtained by adding these six contributions in quadrature. The final result on the pion polarizability is

$$
\alpha_{\pi}=\left(2.0 \pm 0.6_{\text {stat }} \pm 0.7_{\text {syst }}\right) \times 10^{-4} \mathrm{fm}^{3} \text {. }
$$

A measurement with the pion beam replaced by a muon beam of the same momentum was performed in order to validate the result obtained for the pion cross section $d \sigma_{\pi \gamma} / d x_{\gamma}$. The same selection criteria as used for the pion sample are applied adapting the cut $m_{\mu \gamma}<3.5 m_{\mu}$. The simulation for the muon measurement contains the corresponding radiative [15] and form factor corrections. Taking into account the different behavior of the cross section for a pointlike spin- $\frac{1}{2}$ particle, no deviation from the QED prediction is expected for the muon. Using the measurement with the muon beam, the "false polarizability" is determined from the $x_{\gamma}$ dependence of the ratio $R_{\mu}$, that is defined analogously to Eq. (3). It is found to be compatible with zero within statistical uncertainties, $\left(0.5 \pm 0.5_{\text {stat }}\right) \times$ $10^{-4} \mathrm{fm}^{3}$, as shown in the bottom panel of Fig. 3 .

Possible contributions from higher-order polarizabilities beyond Eq. (1), were studied by investigating the sensitivity of the result on the upper limit of $m_{\pi \gamma}$. No significant effect was found when varying this limit between $0.40 \mathrm{GeV} / c^{2}$ and $0.57 \mathrm{GeV} / c^{2}$. Furthermore, the functional behavior of our model, including the chiral-loop corrections, was compared to the approach using dispersion relations [16], and very good agreement was found in the mass range up to $4 m_{\pi}$. The respective cross sections do not differ by more than 2 per mille, which corresponds to less

TABLE I. Estimated systematic uncertainties at $68 \%$ confidence level.

\begin{tabular}{lc}
\hline \hline $\begin{array}{l}\text { Source of } \\
\text { uncertainty }\end{array}$ & $\begin{array}{c}\text { Estimated magnitude } \\
{\left[10^{-4} \mathrm{fm}^{3}\right]}\end{array}$ \\
\hline $\begin{array}{l}\text { Determination of tracking detector } \\
\quad \text { efficiency }\end{array}$ & 0.5 \\
Treatment of radiative corrections & 0.3 \\
Subtraction of $\pi^{0}$ background & 0.2 \\
Strong interaction background & 0.2 \\
Pion-electron elastic scattering & 0.2 \\
Contribution of muons in the beam & 0.05 \\
Quadratic sum & 0.7 \\
\hline \hline
\end{tabular}


than $15 \%$ of the given systematic uncertainty estimate for the polarizability value.

In conclusion, we have determined the pion polarizability from pion Compton scattering embedded in the $\pi^{-} \mathrm{Ni} \rightarrow \pi^{-} \gamma \mathrm{Ni}$ process at small momentum transfer, $Q^{2}<$ $0.0015(\mathrm{GeV} / c)^{2}$. The measurement using a muon beam has revealed no systematic bias of our method. We find the size of the pion polarizability at significant variance with previous experiments and compatible with the expectation from ChPT. This result constitutes important progress towards resolving one of the long-standing issues in low energy QCD.

We gratefully acknowledge the support of the CERN management and staff as well as the skills and efforts of the technicians of the collaborating institutions. This work is supported by MEYS (Czech Republic); "HadronPhysics2" Integrating Activity in FP7 (European Union); CEA, P2I and ANR (France); BMBF, DFG cluster of excellence "Origin and Structure of the Universe", the computing facilities of the Computational Center for Particle and Astrophysics (C2PAP), IAS-TUM and Humboldt foundation (Germany); SAIL (CSR) (India); ISF (Israel); INFN (Italy); MEXT, JSPS, Daiko and Yamada Foundations (Japan); NRF (Republic of Korea); NCN (Poland); FCT (Portugal) and CERN-RFBR (Russia).

\footnotetext{
* Deceased.

${ }^{\dagger}$ Also at Instituto Superior Técnico, Universidade de Lisboa, Lisbon, Portugal.

${ }^{\ddagger}$ Supported by the DFG Research Training Group Programme 1102 "Physics at Hadron Accelerators."
}

${ }^{\S}$ Also at Chubu University, Kasugai, Aichi, 487-8501 Japan. "Also at KEK, 1-1 Oho, Tsukuba, Ibaraki, 305-0801 Japan. "Present address: Universität Bonn, Helmholtz-Institut für Strahlen- und Kernphysik, 53115 Bonn, Germany.

** Also at Moscow Institute of Physics and Technology, Moscow Region, 141700, Russia.

${ }^{\dagger}$ Present address: RWTH Aachen University, III. Physikalisches Institut, 52056 Aachen, Germany.

[1] Y. Antipov, Phys. Lett. 121B, 445 (1983).

[2] J. Ahrens et al. (MAMI A2 Collaboration), Eur. Phys. J. A 23, 113 (2005).

[3] J. Boyer et al. (Mark-II Collaboration), Phys. Rev. D 42, 1350 (1990).

[4] H. Primakoff, Phys. Rev. 81, 899 (1951).

[5] V. A. Petrunkin, Nucl. Phys. 55, 197 (1964).

[6] D. Drechsel and L. V. Filkov, Z. Phys. A 349, 177 (1994).

[7] C. Adolph et al. (COMPASS Collaboration), Phys. Rev. Lett. 108, 192001 (2012).

[8] J. Gasser, M. A. Ivanov, and M. E. Sainio, Nucl. Phys. B745, 84 (2006).

[9] I. Y. Pomeranchuk and I. M. Shmushkevich, Nucl. Phys. 23, 452 (1961).

[10] P. Abbon et al. (COMPASS Collaboration), Nucl. Instrum. Methods Phys. Res., Sect. A 577, 455 (2007).

[11] N. Kaiser and J. M. Friedrich, Nucl. Phys. A812, 186 (2008).

[12] N. Kaiser and J. M. Friedrich, Eur. Phys. J. A 36, 181 (2008).

[13] G. Fäldt, Phys. Rev. C 82, 037603 (2010).

[14] Yu. M. Andreev and E. V. Bugaev, Phys. Rev. D 55, 1233 (1997).

[15] N. Kaiser, Nucl. Phys. A837, 87 (2010); A. B. Arbuzov, J. High Energy Phys. 01 (2008) 031.

[16] B. Pasquini, D. Drechsel, and S. Scherer, Phys. Rev. C 77, 065211 (2008); 81, 029802 (2010); (private communication). 